\title{
Applying the Hierarchy of Controls: What Occupational Safety Can Teach us About Safely Navigating the Next Phase of the Global COVID-19 Pandemic
}

\author{
Neil J. Sehgal ${ }^{1 *}$ and Donald K. Milton ${ }^{2}$ \\ ${ }^{1}$ Department of Health Policy and Management, University of Maryland School of Public Health, College Park, MD, \\ United States, ${ }^{2}$ Maryland Institute for Applied Environmental Health, University of Maryland School of Public Health, \\ College Park, MD, United States
}

OPEN ACCESS

Edited by:

Marc Jean Struelens,

Université libre de Bruxelles, Belgium

Reviewed by:

Liliane Okdah,

King Abdullah International Medical

Research Center (KAIMRC),

Saudi Arabia

Patrick O'Shaughnessy,

The University of lowa, United States David Michaels,

George Washington University, United States

*Correspondence: Neil J. Sehgal sehgal@umd.edu

Specialty section:

This article was submitted to Infectious Diseases - Surveillance,

Prevention and Treatment,

a section of the journal

Frontiers in Public Health

Received: 27 July 2021

Accepted: 11 October 2021

Published: 05 November 2021

Citation:

Sehgal NJ and Milton DK (2021) Applying the Hierarchy of Controls: What Occupational Safety Can Teach us About Safely Navigating the Next

Phase of the Global COVID-19

Pandemic.

Front. Public Health 9:747894

doi: 10.3389/fpubh.2021.747894
Eighteen months into the COVID-19 pandemic, and as the world struggles with global vaccine equity, emerging variants, and the reality that eradication is years away at soonest, we add to notion of "layered defenses" proposing a conceptual model for better understanding the differential applicability and effectiveness of precautions against SARS-CoV-2 transmission. The prevailing adaptation of Reason's Swiss cheese model conceives of all defensive layers as equally protective, when in reality some are more effective than others. Adapting the hierarchy of controls framework from occupational safety provides a better framework for understanding the relative benefit of different hazard control strategies to minimize the spread of SARS-CoV-2.

Keywords: COVID-19, SARS-CoV-2, transmission, hierarchy of controls, layered defenses, layered prevention, PPE

\section{INTRODUCTION}

The recent popularity (1) of the application of James Reason's Swiss cheese model of accident causation to COVID-19 transmission falls short in one important regard-the successive "layers" of defense are too easily perceived as equally effective to control the hazard. A preponderance of evidence now demonstrates that this is not the case $(2,3)$. Inadequacy in defenses and differential risk (e.g., fabric face coverings as compared to filtering respirators, and aerosol "super-spreading" as compared to droplet or fomite transmission) requires a different approach to conceptualizing COVID-19 risk reduction-particularly as COVID-19 continues to devastate the developing world, new and more transmissible variants emerge, vaccines are not yet equitably available across the globe, and eradication of SARS-CoV-2 is decreasingly likely (4). Additionally, framing defensive layers as equally effective may poses challenges to the adoption of more effective mitigation strategies (e.g., vaccination), when the adoption of seemingly equivalent protections may be preferable (5).

\section{THE HIERARCHY OF CONTROLS}

The hierarchy of controls is a framework employed in occupational safety and health to better understand the relative effectiveness of different strategies for risk reduction, and to help determine how to implement feasible and effective solutions (6). The model (Figure 1) is represented 


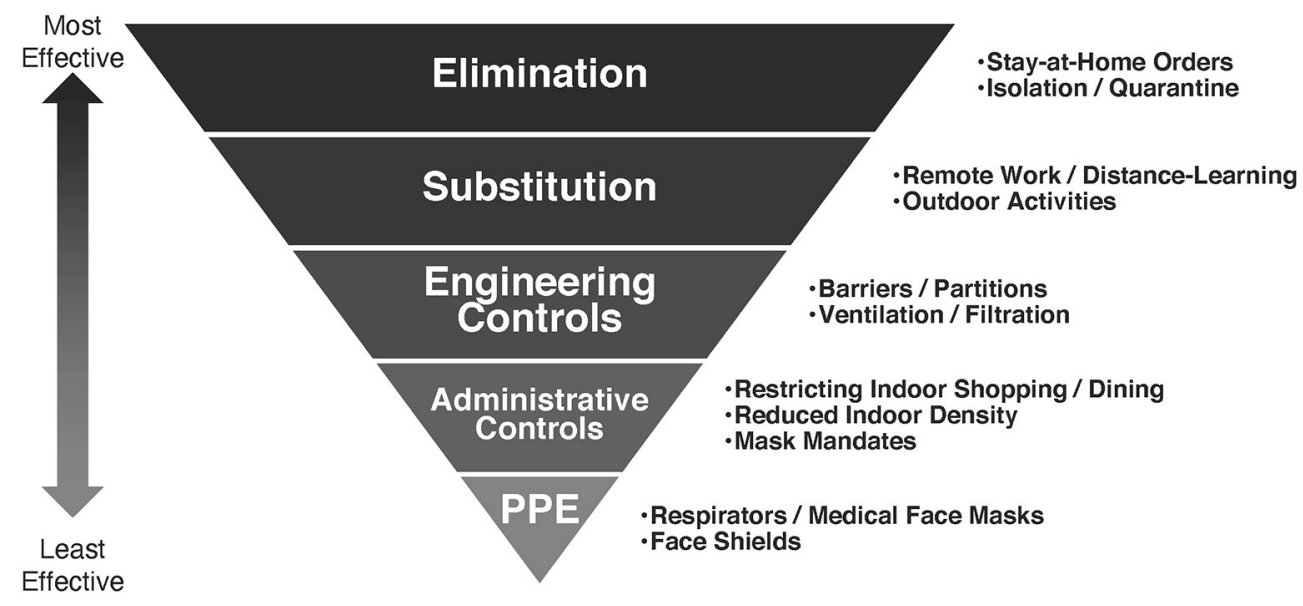

FIGURE 1 | The hierarchy of COVID-19 controls.

as an upside-down pyramid, with five categories represented in descending order of effectiveness: elimination, substitution, engineering controls, administrative controls, and personal protective equipment (PPE). And, while developed to better manage exposures to occupational hazards and protect workers, the model has broad applicability in helping healthcare workers, policy makers, and the public better understand the relative effectiveness of strategies to prevent the transmission of an airborne infectious virus like SARS-CoV-2, and the paradigm is increasingly being adopted to conceptualize COVID-19 risk reduction $(7,8)$. The fundamental idea behind the hierarchy is that, while different hazard controls are effective at minimizing risk, those at the top of the model are more protective than those at the bottom. As in occupational safety and health, employing the most effective methods first and most frequently can best minimize the risk of COVID-19 transmission.

Recent revisions to Centers for Disease Control and Prevention guidance on transmission notes "infections with respiratory viruses are principally transmitted through three modes: contact, droplet, and airborne." (9) Contact or "fomite" transmission occurs through touching an infectious person or a surface or item that is contaminated with virus. Droplet transmission occurs through exposure to virus-containing respiratory droplets directly from an infectious person to a susceptible person at close range. Airborne transmission occurs through exposure to smaller virus-containing droplets and particles that float in the air, are highly concentrated close to the person who exhaled them and can remain suspended in air for many seconds to hours and accumulate in the air of poorly ventilated spaces. A key weakness in early COVID-19 response was in treating these hazards as equivalent; recent research has demonstrated comparatively low risk of contact transmission (10), and the World Health Organization has noted that despite evidence of the survival of SARS-CoV-2 on certain surfaces, no reports have directly demonstrated fomite transmission (11). Emerging evidence, on the other hand, suggests significant risk associated with close-contact transmission over short distances and in poorly ventilated spaces, with airborne transmission making the largest contribution $(12,13)$. Though protecting against each of these hazards is necessary, currently employed controls are not equally effective against each, and higher-order controls should be prioritized.

\section{Elimination and Substitution}

Elimination and substitution, the most effective hazard controls, involve physically removing the hazard and associated risk or substituting the hazard with something less risky. Stay-athome orders employed early in the pandemic were attempts to eliminate transmission risk in communities. While in principle effective, the dire social, psychological, and economic impacts of "lock-downs" limits their long-term viability and necessitate that they be used only briefly while other measures are put in place. Isolation and quarantine are additional means of eliminating transmission risk from infected or potentially infectious individuals. As a precautionary measure, eliminating unnecessary public outings or gatherings is another effective means of removing the risk of SARS-CoV-2 infection. Similarly, allowing remote work or moving indoor-activities outdoors substitutes the hazards incumbent in in-person activities with less risky alternatives. Vaccines against COVID-19 have proven to be a very effective pharmacologic elimination strategy where available.

\section{Engineering Controls}

Engineering controls do not eliminate hazards, but rather isolate individuals from them. Physical barriers separating individuals who must interact at close range are a now very common example of an engineering control. Importantly, however, as they do not eliminate hazards engineering controls must be employed in concert with other controls. Improving the safety of indoor air by increasing ventilation and filtration or employing upperroom germicidal ultraviolet light are as-yet underemployed engineering controls which can further minimize COVID-19 transmission risk $(14,15)$. Well-designed engineering controls 
can be highly effective, reducing risk to individuals independent of their own behaviors, and can significantly enhance protection for individuals adherent to administrative controls or employing PPE (16). A key limitation of engineering controls as a community mitigation strategy is that, unlike in industry, it is not always possible to redesign indoor spaces, ventilation systems, and other infrastructure to sufficiently ameliorate the risk of pathogenic transmission. Where engineering controls are able to limit concentrations of indoor respiratory aerosols, however, they are effective in reducing "far-field" airborne transmission of infectious agents like SARS-CoV-2.

\section{Administrative Controls}

Administrative controls involve changing individual behaviors via policy or mandate to minimize hazard risk, and are the control most frequently instituted to increase social distancing, and to reduce person-to-person interaction or population density in defined spaces to protect against COVID-19 infection. Policies restricting indoor activities like large gatherings or dining, and organizational-level mandates like remote-work, staggered inperson work schedules, or distance learning are administrative means of minimizing density-attributed risk. Vaccine and maskmandates are another administrative control, though their effectiveness has been limited by social and political resistance. This illustrates the key weakness of reliance on administrative controls (and the challenge in relying exclusively on them to mitigate transmission risk); compliance is necessary for administrative controls to be effective, and even well-intentioned individuals are prone to slips and lapses in adherence. And, as lessons from patient safety and risk management teach, policies that rely on perfect adherence are inadequate and doomed to fail.

\section{Personal Protective Equipment}

The final risk reduction strategy is the employment of PPE, protecting individuals from known hazard exposure using respirators, eye protection, and other individually donned protective items. PPE can reduce risk of hazard and its use has been an essential strategy to limit COVID-19 transmission,

\section{REFERENCES}

1. Roberts S. The swiss cheese model of pandemic defense. The New York Times (2020, June 30). Available online at: https://www.nytimes.com/2020/12/05/ health/coronavirus-swiss-cheese-infection-mackay.html.

2. Chu DK, Akl EA, Duda S, Solo K, Yaacoub S, Schünemann HJ, et al. Physical distancing, face masks, and eye protection to prevent person-to-person transmission of SARS-CoV-2 and COVID-19: a systematic review and metaanalysis. Lancet. (2020) 395:1973-87. doi: 10.1016/S0140-6736(20)31142-9

3. Shen M, Zu J, Fairley CK, Pagán JA, An L, Du Z, et al. Projected COVID-19 epidemic in the United States in the context of the effectiveness of a potential vaccine and implications for social distancing and face mask use. Vaccine. (2021) 39:2295-302. doi: 10.1016/j.vaccine.2021.02.056

4. Murray CJL, Piot P. The potential future of the COVID-19 pandemic: will SARS-CoV-2 become a recurrent seasonal infection? JAMA. (2021) 325:1249. doi: 10.1001/jama.2021.2828

5. Trogen B, Caplan A. Risk compensation and COVID-19 vaccines. Ann Intern Med. (2021) 174:858-9. doi: 10.7326/M20-8251 though it is necessarily less protective than controls higher in the hierarchy. The effectiveness of PPE as a control is additionally limited as it is reliant on both adequate supply and proper and continuous use. In circumstances where hazards are truly unavoidable, PPE use is critical. In work-settings reliant on PPE for hazard control, regulations mandate additional controls as well. Lay-persons are infrequently trained in the correct use of PPE, and in the current pandemic frequently ill-equipped-nonmedical masks and fabric face coverings are not PPE, and wearing a non-medical mask does not eliminate SARS-CoV-2 infection risk to the individual (17).

\section{DISCUSSION}

As it is increasingly likely that COVID-19 will remain endemic, as more transmissible variants emerge, and as at our current pace we may not vaccinate people in low-income countries until the end of 2022 or beyond (18), our continued reliance on the least effective controls and our continued treatment of controls as substitutes instead of complements-such as reliance on face-coverings or social distancing, but not both-has limited our ability to keep SARS-CoV2 in-check. The hierarchy of controls is an effective model for understanding both the relative effectiveness of hazard minimization, and that while most strategies are necessary few are sufficient to slow the spread of COVID-19.

\section{DATA AVAILABILITY STATEMENT}

The original contributions presented in the study are included in the article/supplementary material, further inquiries can be directed to the corresponding author.

\section{AUTHOR CONTRIBUTIONS}

NS conceptualized the piece and drafted the first version of the manuscript. NS and DM commented on and edited the draft. Both authors reviewed and agreed with the final version.

6. The National Institute for Occupational Safety and Health. Hierarchy of Controls. Available online at: https://www.cdc.gov/niosh/topics/hierarchy/ default.html (accessed October 1, 2021)

7. The National Institute for Occupational Safety and Health, Department of Labor. Guidance on Preparing Workplaces for COVID-19 (OSHA 3990-02 2020). Available online at: https://www.osha.gov/sites/default/files/ publications/OSHA3990.pdf (accessed October 1, 2021)

8. American Society of Safety Professionals. How to Apply the Hierarchy of Controls in a Pandemic. Available online at: https://www.assp.org/news-andarticles/how-to-apply-the-hierarchy-of-controls-in-a-pandemic (accessed October 1, 2021)

9. CDC. Coronavirus Disease 2019 (COVID-19). Centers for Disease Control and Prevention. (2020). Available online at: https://www.cdc.gov/coronavirus/ 2019-ncov/science/science-briefs/sars-cov-2-transmission.html (accessed June 30, 2021)

10. Mondelli MU, Colaneri M, Seminari EM, Baldanti F, Bruno R. Low risk of SARS-CoV-2 transmission by fomites in real-life conditions. Lancet Infect Dis. (2021) 21:e112. doi: 10.1016/S1473-3099(20)30678-2 
11. Transmission of SARS-CoV-2: Implications for Infection Prevention Precautions. Available online at: https://www.who.int/news-room/ commentaries/detail/transmission-of-sars-cov-2-implications-forinfection-prevention-precautions (accessed June 30, 2021)

12. Hamner L. High SARS-CoV-2 attack rate following exposure at a choir practice-Skagit County, Washington, march 2020. MMWR Morb Mortal Wkly Rep. (2020) 69:606-10. doi: 10.15585/mmwr.mm6919e6

13. Jang S, Han SH, Rhee J-Y. Cluster of coronavirus disease associated with fitness dance classes, South Korea. Emerg Infect Dis. (2020) 26:1917-20. doi: 10.3201/eid2608.200633

14. Morawska L, Milton DK. It is time to address airborne transmission of coronavirus disease 2019 (COVID-19). Clin Infect Dis. (2020) 71:2311-3. doi: $10.1093 / \mathrm{cid} /$ ciaa939

15. Morawska L, Allen J, Bahnfleth W, Bluyssen PM, Boerstra A, Buonanno G, et al. A paradigm shift to combat indoor respiratory infection. Science. (2021) 372:689-91. doi: 10.1126/science.abg2025

16. Allen JG, Ibrahim AM. Indoor air changes and potential implications for SARS-CoV-2 transmission. JAMA. (2021) 325:2112. doi: 10.1001/jama.2021.5053

17. Gandhi M, Marr LC. Uniting infectious disease and physical science principles on the importance of face masks for
COVID-19.
Med.
$(2021)$
2:29-32. doi:
10.1016/j.medj.2020

12.008

18. Irwin A. What it will take to vaccinate the world against COVID-19. Nature (2021) 592:176-8. doi: 10.1038/d41586-021-00727-3

Conflict of Interest: The authors declare that the research was conducted in the absence of any commercial or financial relationships that could be construed as a potential conflict of interest.

Publisher's Note: All claims expressed in this article are solely those of the authors and do not necessarily represent those of their affiliated organizations, or those of the publisher, the editors and the reviewers. Any product that may be evaluated in this article, or claim that may be made by its manufacturer, is not guaranteed or endorsed by the publisher.

Copyright (๑) 2021 Sehgal and Milton. This is an open-access article distributed under the terms of the Creative Commons Attribution License (CC BY). The use, distribution or reproduction in other forums is permitted, provided the original author(s) and the copyright owner(s) are credited and that the original publication in this journal is cited, in accordance with accepted academic practice. No use, distribution or reproduction is permitted which does not comply with these terms. 